\title{
Study of factors affecting resolution of urinary tract infection following treatment of constipation in Iranian children who visited a tertiary referral hospital
}

\author{
Farid Imanzadeh ${ }^{1}$, Ali-Akbar Sayyari ${ }^{1}$, Mostafa Sharifian ${ }^{1}$, Hazhir Javaherizadeh², Parisa Aghasi ${ }^{1}$ \\ ${ }^{1}$ Mofid Children's Hospital, Shahid Beheshti University of Medical Sciences, Tehran, Iran \\ ${ }^{2}$ Ahvaz Jundishapur University of Medical Sciences, Ahvaz, Iran
}

Przegląd Gastroenterologiczny 2012; 7 (2): 78-80

DOI: $10.5114 / p g .2012 .28648$

Key words: constipation, urinary tract infection, children.

Address for correspondence: Hazhir Javaherizadeh MD, Ahvaz Jundishapur University of Medical Sciences, Ahvaz, Iran, phone/fax:+98-631-3331031/3335363, e-mail: hazhirja@yahoo.com

\begin{abstract}
Aim: The aim of this study was to evaluate the effect of sex, age, number of urinary tract infections, and duration of constipation on resolution of urinary tract infection (UTI) following treatment of constipation in Iranian children referred to pediatric gastroenterology and pediatric nephrology clinics.

Material and methods: This study was conducted in Mofid Children's Hospital. Duration of the study was from 1 October 2010 to 31 June 2011. In this study every child who visited due to urinary tract infection or constipation was asked about the other condition. Data were analyzed by SPSS ver. 16.0 (Chicago, IL, USA). $\chi^{2}$ and $t$-test were used for comparison.

Results: In this study, 550 children visited the hospital due to urinary tract infection or constipation. Finally 380 patients
\end{abstract}

\section{Introduction}

Constipation is one of the most common digestive disorders around the world [1]. Several studies suggest that constipation plays an etiologic role in urinary tract abnormalities [2-4]. We have observed many cases without treatment of this etiologic factor. However, there have been published studies that show a correlation between constipation and urinary tract abnormalities, but there is limited research on the factors affecting treatment of urinary tract infection following treatment of constipation.

\begin{abstract}
Aim
The aim of this study was to evaluate the effect of sex, age, number of urinary tract infections (UTI), and sex on resolution of urinary tract infection following treatment of constipation in Iranian children referred to pediatric gastroenterology and pediatric nephrology clinics.
\end{abstract}

with constipation and UTI were detected. Among 220 patients with UTI > 2 times, 90 (40.90\%) showed UTI resolution after treatment of constipation. Of 160 patients with $\leq 2$ UTIs, 85 (53.12\%) showed UTI improvement following treatment of constipation $(p=0.018)$. In children younger than 1 year, treatment of UTI after treatment of constipation was more effective than in other ages $(p<0.001)$. There was no significant difference between males and females. In children with constipation duration less than 6 months, most cases of urinary tract infection resolved after treatment of constipation $(p<0.001)$.

Conclusions: The UTI resolution after treatment of constipation was higher in children $<1$ year old. If the patient has a history of more than 2 UTIs, improvement of UTI following treatment of constipation will be decreased.

\section{Material and methods}

This study was conducted in Mofid Children's Hospital. Duration of the study was from 1 October 2010 to 31 June 2011. In this study every child who visited due to urinary tract infection or constipation was asked about the other condition. Data were analyzed by SPSS ver. 13.0 (Chicago, IL, USA). $\chi^{2}$ and $t$-test were used for comparison.

\section{Results}

In this study, 550 children visited the hospital with urinary tract infection or constipation. Of these cases, 165 cases had no history of UTI. Finally 380 patients with constipation and a history of urinary tract infection were detected. Of these 380 patients, 160 had UTI 2 times or less and 220 cases had UTI more than two times. 
The UTI resolution following treatment of constipation was significantly higher in cases with history of UTI $\leq 2$ times (Table I) $(p=0.018)$. In patients less than 1 year old, treatment of UTI after treatment of constipation was more effective than in other ages $(p<0.001)$ (Table II). The results showed no significant difference between males and females (Table III).

In children with constipation duration less than 6 months, most cases of urinary tract infection resolved after treatment of constipation ( $p<0.001$ ) (Table IV).

Of 380 patients, 275 had normal urine analysis and 105 had abnormal urine analysis. Of 275 patients with normal urine analysis, 155 (56.36\%) had UTI resolution following treatment of constipation. From 105 patients with abnormal urine analysis, 20 (19.40\%) showed UTI improvement following treatment of constipation. There was a significant difference between the two groups $(p<0.001)$.

\section{Discussion}

Of 550 patients with constipation or UTI, 380 (69.09\%) had both conditions. Neumann et al. studied 131 pediatric patients with UTI. They found that $34 \%$ of children presenting for UTI had abnormal bowel habits [5]. The rate of abnormal bowel habits among UTI cases in our study was higher.

Romanczuk and Korczawski in a prospective study with physical testing and treatment intervention found that recurrent UTI was present in 39\% of children hospitalized for chronic constipation [2]. This high rate of constipation associated with urinary tract infection in our cases showed failure of detection by the primary physician or parent's unawareness about constipation. Loening-Baucke reported disappearance of urinary tract infection following treatment of constipation in cases without urinary tract abnormality [3]. O'Regan et al. studied 47 pediatric cases with recurrent UTI. They found that children with recurrent UTI have large fecal reservoirs by rectal examination and manometry [6].

In a retrospective case control chart review by Blethyn et al., on 61 pediatric patients who referred for investigation of UTI, a significant association between fecal loading and number of UTIs was detected [7].

Halachami and Farhat in their review article stated that adjunctive treatment of constipation and voiding dysfunction is important [8]. But in their review, the role of age, sex, and duration of constipation was unclear.

\section{Conclusions}

In children less than 1 year old, UTI resolution following treatment of constipation was higher than in other age groups. With advancing age, treatment of con-
Table I. Effect of number of UTIs on its resolution following constipation

\begin{tabular}{lccc} 
Number of infections & Yes (\%) & No (\%) & Total \\
\hline Two or less & $85(53.12)$ & $75(46.88)$ & $160(100)$ \\
\hline More than two & $90(40.90)$ & $130(59.10)$ & $220(100)$ \\
$\quad p=0.018$ & & &
\end{tabular}

Table II. UTI resolution after treatment of constipation among different age groups

\begin{tabular}{lccc} 
Age & Yes $(\%)$ & No $(\%)$ & Total $(\%)$ \\
\hline$<1$ year & $85(68)$ & $40(32)$ & $125(100)$ \\
\hline $1-5$ years & $90(66.67)$ & $45(33.33)$ & $135(100)$ \\
\hline $5-9$ years & 0 & $20(100)$ & $20(100)$ \\
$p<0.001$ & & &
\end{tabular}

Table III. UTI resolution following treatment of constipation in both sexes

\begin{tabular}{lccc} 
Sex & Yes (\%) & No (\%) & Total (\%) \\
\hline Male & $90(43.90)$ & $115(56.10)$ & $205(100)$ \\
\hline Female & $85(48.57)$ & $90(51.43)$ & $175(100)$ \\
$\quad p=0.409$ & & &
\end{tabular}

Table IV. Correlation of duration of constipation and UTI resolution following treatment of constipation

\begin{tabular}{lccc}
\hline Duration of constipation & Yes (\%) & No (\%) & Total (\%) \\
\hline Less than 6 months & $85(70.83)$ & $35(29.17)$ & $120(100)$ \\
\hline 6 months - 1 year & 0 & $60(100)$ & $60(100)$ \\
\hline More than 1 year & $90(45)$ & $110(55)$ & $200(100)$ \\
$\quad p<0.001$ & & & \\
& & &
\end{tabular}

stipation has less effect on UTI resolution. We recommend early diagnosis and treatment of constipation to achieve better results in treatment of UTI.

The limitation in this study was the retrospective study method.

\section{References}

1. Higgins PD, Johanson JF. Epidemiology of constipation in North America: a systematic review. Am J Gastroenterol 2004; 99: 750-9.

2. Romanczuk W, Korczawski R. Chronic constipation: a cause of recurrent urinary tract infections. Turk J Pediatr 1993; 35: 181-8.

3. Loening-Baucke $V$. Urinary incontinence and urinary tract infection and their resolution with treatment of chronic constipation of childhood. Pediatrics 1997; 100: 228-32.

4. Loening-Baucke V. Prevalence rates for constipation and faecal and urinary incontinence. Arch Dis Child 2007; 92: 486-9. 
5. Neumann PZ, DeDomenico IJ, Nogrady MB. Constipation and urinary tract infection. Pediatrics 1973; 52: 241-5.

6. O’Regan S, Yazbeck S, Schick E. Constipation, bladder instability, urinary tract infection syndrome. Clin Nephrol 1985; 23: 152-4.

7. Blethyn AJ, Jenknis HR, Roberts R, et al. Radiological evidence of constipation in urinary tract infection. Arch Dis Child 1995; 73: 534-5.

8. Halachmi S, Farhat WA. Interactions of constipation, dysfunctional elimination syndrome, and vesicourethral reflux. Adv Urol 2008; 828275. 